\title{
Códigos Perfeitos Bidimensionais em Reticulados Algébricos
}

\author{
João E. Strapasson ${ }^{1}$ \\ Unicamp, Limeira, SP \\ Giselle Strey ${ }^{2}$ \\ Unicamp, Campinas, SP
}

\begin{abstract}
Resumo. Este trabalho consiste numa investigação sobre a quantidade de Códigos Perfeitos, subreticulados, em Reticulados Algébricos bidimensionais obtidos via mergulho de Minkowski. Em contraste com os Códigos Perfeitos nos reticulados $\mathbb{Z}^{2}$ e Hexagonal, considerar os Reticulados Algébricos resultou em um número grande de Códigos Perfeitos.
\end{abstract}

Palavras-chave. Reticulados Algébricos, Código Perfeitos, Mergulho de Minkowski.

\section{Introdução}

Ao longo dos últimos anos, diversos autores exploraram a existência e a não existência de Códigos Perfeitos (no reticulado $\mathbb{Z}^{n}$ ), vide $[4,6,7,9,10]$. Em especial os autores Golomb and Welch, em [5], conjecturam que na métrica $\ell_{1}$ (métrica do táxi) os perfeitos existiam para dimensões baixas e raios pequenos. E, e então diversos autores deram suas contribuições reforçando a conjectura.

Constata-se que há poucos Códigos Perfeitos no ambiente $\mathbb{Z}^{n}$, quando se leva em conta as métricas: do táxi e a métrica euclidiana. Então outros autores começaram a explorar as métricas $\ell_{p}$ [1], e também o estudo de Códigos Quase-perfeitos [11]. Mesmo considerando essas outras métricas o número de códigos Perfeitos, ainda aparecem em números modestos, e também restritos às dimensões baixas.

Há ainda autores que investigaram um pouco sobre Códigos Perfeitos sob alguma métrica no reticulado raiz $A_{n}$, (vide $[3,8]$ ), e inspirados nestes estudos, passamos a investigar e estabelecer condições de necessárias para a existência destes códigos em reticulados mais gerais, i.e., não restritos apenas aos reticulados $Z^{n}$ e $A^{n}$. Este reticulado mais geral será a partir deste momento denominado de Reticulado Ambiente, conceito introduzido em [12].

A condição de existência de perfeitos está fortemente atrelada ao raio de cobertura do reticulado ambiente. Em outras palavras, o reticulado ambiente que possui um raio de cobertura grande tem muito mais chances de possuir Códigos Perfeitos e em maior quantidade.

Neste trabalho consideraremos em especial duas famílias como reticulado ambiente, tais famílias foram obtidas via mergulho de Minkowski, e cuja matriz de Gram, que é integral, depende de um parâmetro inteiro $l$, a medida que aumentamos o parâmetro $l$, o raio de cobertura do reticulado ambiente aumenta, e o número de Códigos Perfeitos também aumenta.

Este trabalho está subdividido nas seguintes seções: Preliminares (nesta seção iremos introduzir os elementos essenciais deste trabalho), Códigos Perfeitos (aqui generalizamos as condições de existência para reticulados ambientes gerais), e Códigos Perfeitos em Reticulados Algébricos Bidimensionais (nesta última faremos um estudo de caso, em que o número de Códigos Perfeitos pode ser muito grande).

\footnotetext{
${ }^{1}$ strapass@unicamp.br.

2 giselleribeiro2006@hotmail.com.
} 


\section{Preliminares}

Nesta seção vamos introduzir alguns conceitos básicos, limitados aos essenciais a este trabalho, mais detalhes vide [2].

\section{$2.1 \quad$ Reticulados}

Seja $\left\{\boldsymbol{v}_{1}, \ldots, \boldsymbol{v}_{m}\right\}$ um conjunto de vetores linearmente independentes em $\mathbb{R}^{n}$. O conjunto

$$
\Lambda=\left\{\sum_{i=1}^{m} \lambda_{i} \boldsymbol{v}_{i}: \lambda_{i} \in \mathbb{Z}, \forall i \in\{1, \ldots, m\}\right\}
$$

é chamado de reticulado e $\left\{\boldsymbol{v}_{1}, \ldots, \boldsymbol{v}_{m}\right\}$ é dita uma base do reticulado. Neste caso, dizemos que o reticulado $\Lambda$ é gerado pela base $\left\{\boldsymbol{v}_{1}, \ldots, \boldsymbol{v}_{m}\right\}$.

O espaço gerado por todas as combinações lineares dos vetores $v_{1}, \ldots, v_{n}$ é denotado por $\operatorname{span}\left\{v_{1}, \ldots, v_{n}\right\}$. Se $M$ é uma matriz geradora para um reticulado $\Lambda$, representamos por $\operatorname{span}(M)$ ou $\operatorname{span}(\Lambda)$ o espaço vetorial gerado pelas linhas da matriz $M$.

Seja $\Lambda \subset \mathbb{R}^{n}$ um reticulado e $\left\{\boldsymbol{v}_{1}, \ldots, \boldsymbol{v}_{m}\right\}$ uma base de $\Lambda$, em que $\boldsymbol{v}_{i}=\left(v_{i 1}, \ldots, v_{i n}\right)$ para $i \in\{1, \ldots, m\}$. Chamamos de matriz geradora de $\Lambda$ a matriz $M$ cujas linhas são formadas pelos vetores de uma base do reticulado, isto é,

$$
M=\left(\begin{array}{ccc}
v_{11} & \ldots & v_{1 n} \\
\vdots & \ddots & \vdots \\
v_{m 1} & \cdots & v_{m n}
\end{array}\right)
$$

é uma matriz geradora de $\Lambda$.

Dois reticulados $\Lambda_{1}$ e $\Lambda_{2}$ em $\mathbb{R}^{n}$ são ditos equivalentes (e denotados por $\Lambda_{1} \sim \Lambda_{2}$ ) se existirem uma isometria $\sigma: \mathbb{R}^{n} \longrightarrow \mathbb{R}^{n}$ e um número real positivo $c$ tais que $(c \sigma)\left(\Lambda_{1}\right)=\Lambda_{2}$. Dizemos que $c$ é a razão de semelhança de $\Lambda_{1}$ para $\Lambda_{2}$.

A matriz $G=M M^{T}$ é chamada de matriz de Gram do reticulado gerado por $M$. O determinante do reticulado $\Lambda$, denotado por $\operatorname{det}(\Lambda)$, é definido como o determinante da matriz de $\operatorname{Gram}$, isto é, $\operatorname{det}(\Lambda)=\operatorname{det}(G)$.

Dizemos que $\Lambda_{1} \subset \Lambda$ é um sub-reticulado de $\Lambda$ se $\Lambda_{1}$ também for um reticulado.

Um reticulado $\Lambda \subset \mathbb{R}^{n}$ é dito integral se $\boldsymbol{x} \cdot \boldsymbol{y} \in \mathbb{Z}$, para quaisquer $\boldsymbol{x}, \boldsymbol{y} \in \Lambda$.

Sejam $\Lambda \subset \mathbb{R}^{n}$ um reticulado e $\beta=\left\{b_{1}, \ldots, b_{m}\right\}$, com $m \leq n$, uma base para $\Lambda$. Dado um elemento $\boldsymbol{v}$ de $\Lambda$, a região de Voronoi de $\boldsymbol{v}$ é definida como

$$
\mathcal{V}(\boldsymbol{v})=\{\boldsymbol{x} \in \operatorname{span}(\beta):\|\boldsymbol{x}-\boldsymbol{v}\| \leq\|\boldsymbol{x}-\boldsymbol{u}\|, \forall \boldsymbol{u} \in \Lambda\} .
$$

Em outras palavras, a região de Voronoi de $\boldsymbol{v}$ é formada por todos os pontos do span( $\beta$ ) que estão mais próximos ou a mesma distância de $\boldsymbol{v}$ do que de qualquer outro ponto de $\Lambda$.

\subsection{Empacotamento e Cobertura}

Seja $V$ um espaço vetorial normado. A bola fechada (real) centrada em $\boldsymbol{z} \in V$ com raio $r>0$, denotada por $B(\boldsymbol{z}, r)$, é definida como $B(\boldsymbol{z}, r)=\left\{\boldsymbol{x} \in V:\|\boldsymbol{x}-\boldsymbol{z}\| \leq r^{2}\right\}$. Quando $\boldsymbol{z}=\mathbf{0}$, por simplicidade, denotaremos a bola como $B_{r}$ ao invés de $B(\mathbf{0}, r)$.

Um empacotamento esférico, ou simplesmente um empacotamento em $V$, é uma distribuição de bolas/esferas, todas de mesmo raio, de forma que duas bolas/esferas não se interceptam ou se interceptam apenas nos bordos. Um empacotamento reticulado é um empacotamento esférico em que o conjunto dos centros das bolas/esferas forma um reticulado em $V$. 
O raio de empacotamento de um reticulado $\Lambda \subset \mathbb{R}^{n}$, denotado por $\rho$, é o maior raio para o qual é possível distribuir bolas/esferas centradas nos elementos de $\Lambda$ e obter um empacotamento reticulado, isto é,

$$
\rho=\max \left\{r \in \mathbb{R}: \Lambda+B_{r}=\mathbb{R}^{n}\right\}
$$

é raio de empacotamento.

Dado um reticulado $\Lambda \subset \mathbb{R}^{n}$, definimos a densidade de empacotamento esférico de $\Lambda$ como

$$
\begin{aligned}
\Delta(\Lambda) & =\frac{\text { volume de uma esfera de raio } \rho}{\operatorname{volume~da~região~fundamental~de~} \Lambda}= \\
& =\frac{\operatorname{vol}\left(B_{\rho}\right)}{\operatorname{vol}(\Lambda)}=\frac{\operatorname{vol}\left(B_{1}\right) \rho^{n}}{\operatorname{vol}(\Lambda)}=\frac{\operatorname{vol}\left(B_{1}\right) \rho^{n}}{\operatorname{det}(\Lambda)^{1 / 2}} .
\end{aligned}
$$

Sejam $\Lambda \subset \mathbb{R}^{n}$ um reticulado e $V=\operatorname{span}(\Lambda)$, dizemos que o conjunto $\Lambda+B_{r}$ é uma cobertura de $V$ se $V \subset\left(\Lambda+B_{r}\right)$.

Sejam $\Lambda \subset \mathbb{R}^{n}$ um reticulado e $V=\operatorname{span}(\Lambda)$, o número

$$
R_{\Lambda}=\min \left\{r: \Lambda+B_{r} \text { é uma cobertura do } V\right\}
$$

é chamado de raio de cobertura de $\Lambda$. Em outras palavras, o raio de cobertura de um reticulado $\Lambda$ é o menor $r>0$ tal que $\bigcup_{\boldsymbol{z} \in \Lambda} B(\boldsymbol{z}, r) \supset V$.

Sejam $\Lambda$ um reticulado e $V=\operatorname{span}(\Lambda)$, a densidade de cobertura em $V$ de $\Lambda$ é dada por

$$
\Theta(\Lambda)=\frac{V_{n} R_{\Lambda}}{\operatorname{vol}(\Lambda)}
$$

\section{Códigos Perfeitos}

Dado um reticulado $\Lambda_{a}$, a bola discreta, denotada por $\tilde{B}(\boldsymbol{z}, r)$, centrada em $\boldsymbol{z}=\left(z_{1}, \ldots, z_{n}\right) \in$ $\Lambda_{a}$ e com raio $r$, é definida como $\tilde{B}(\boldsymbol{z}, r)=\left\{\left(x_{1}, \ldots, x_{n}\right) \in \Lambda_{a}:\left(x_{1}-z_{1}\right)^{2}+\cdots+\left(x_{n}-z_{n}\right)^{2} \leq r^{2}\right\}$. Quando $\boldsymbol{z}=\mathbf{0}$, por simplicidade escreveremos $\tilde{B}_{r}$ ao invés de $\tilde{B}(\mathbf{0}, r)$.

Seja $\Lambda \subset \Lambda_{a}$ um reticulado. Como é bem conhecido na literatura, a distância mínima de um reticulado $\Lambda$, denotada por $d(\Lambda)$, é a norma euclidiana de um vetor não-nulo de norma mínima de $\Lambda$, e o raio de empacotamento, denotado por $r(\Lambda)$, é definido como o maior $r$ tal que

(i) $\left(\left(\tilde{B}_{r}+\boldsymbol{\lambda}\right) \cap \tilde{B}_{r}\right) \cap \Lambda_{a}=\emptyset$, em que $\mathbf{0} \neq \boldsymbol{\lambda} \in \Lambda$;

(ii) $\left[q^{r^{2}}\right] \Theta_{\Lambda_{a}} \neq 0$, isto é, existem pontos do reticulado com norma igual a $r$.

Se, além das duas propriedades acima, $\Lambda$ cumprir a seguinte propriedade:

(iii) $\tilde{B}_{r}+\Lambda=\Lambda_{a}$;

dizemos que $\Lambda$ é um código $r$-perfeito em $\Lambda_{a}$. Vale ressaltar que neste capítulo estaremos denotando reticulado por código.

Seja $\mathcal{D}_{n}$ o conjunto de todas as distâncias atingíveis em $\Lambda_{a}$, isto é,

$$
\mathcal{D}_{n}=\mathcal{D}_{n}\left(\Lambda_{a}\right)=\left\{\|\boldsymbol{x}\|: \boldsymbol{x} \in \Lambda_{a}\right\} .
$$

Considere a seguinte aplicação

$$
\begin{aligned}
\Phi: \mathbb{R} & \longrightarrow \mathcal{D}_{n} \\
x & \longmapsto \max \left\{y \in \mathcal{D}_{n}: y \leq x\right\} .
\end{aligned}
$$


Definimos anteriormente o raio de cobertura de um código (reticulado) $\Lambda \subset \mathbb{R}^{n}$, que é o menor número real $r$ tal que $\bigcup_{\boldsymbol{z} \in \Lambda} \tilde{B}(\boldsymbol{z}, r) \supset \mathbb{R}^{n}$. Agora, vamos definir o raio de cobertura discreto de um código.

O raio de cobertura (discreto) de um código $\Lambda$, denotado por $\tilde{R}_{\Lambda}$, é o menor $r \in \mathcal{D}_{n}$ tal que $\bigcup_{z \in \Lambda} \tilde{B}(\boldsymbol{z}, r)=\Lambda_{a}$.

\subsection{Condições de Existência}

Teorema 3.1. Em um espaço ambiente $\Lambda_{a}$, o raio de um código linear perfeito $\Lambda$ satisfaz

$$
\begin{aligned}
r & \leq M \frac{\left(1+\left(\Delta^{n}\right)^{1 / n}\right)}{\left(1-\left(\Delta^{n}\right)^{1 / n}\right)} \\
& \leq R_{\Lambda_{a}} \frac{\left(1+\left(\Delta^{n}\right)^{1 / n}\right)}{\left(1-\left(\Delta^{n}\right)^{1 / n}\right)}
\end{aligned}
$$

em que $\Delta^{n}$ é a densidade máxima de um reticulado de posto completo, $M=\min _{\boldsymbol{z} \in \Lambda_{a}}\|\boldsymbol{x} / 2-\boldsymbol{u}\| e$ $\boldsymbol{x}$ é um vetor de norma mínima em $\Lambda$.

Teorema 3.2. Em um espaço ambiente $\Lambda_{a}$, o raio $r$ de um código linear perfeito $\Lambda_{r}$ satisfaz

$$
r \leq R_{\Lambda_{a}} \frac{\left(\left(\Theta^{n}\right)^{1 / n}+1\right)}{\left(\left(\Theta^{n}\right)^{1 / n}-1\right)}
$$

em que $\Theta^{n}$ é o ínfimo das densidades de cobertura sobre todos os reticulados $n$-dimensionais de posto completo e $R_{\Lambda_{a}}$ é o raio de cobertura de $\Lambda_{a}$.

\section{Códigos Perfeitos em Reticulados Algébricos Bidimensi- onais}

Neste trabalho vamos considerar duas famílias de reticulados algébricos bidimensionais, obtidas pelo mergulho de Minkowski, o processo de obtenção destes reticulados será omitido aqui:

\section{1 $-l \equiv 1(\bmod 4)$}

O reticulado algébrico $\Lambda_{a, l, 1}$ possui matrizes geradora e de Gram, respectivamente:

$$
M_{l}=\left(\begin{array}{cc}
\sqrt{2} & 0 \\
\sqrt{2} / 2 & \sqrt{2 l} / 2
\end{array}\right) \text { e } G_{l}=\left(\begin{array}{cc}
2 & 1 \\
1 & (1+l) / 2
\end{array}\right) .
$$

\subsubsection{Tipo 1: polihexágono de um nível}

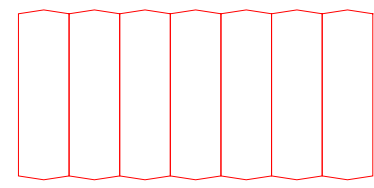

Figura 1: Polihexágono do Tipo 1 construído para $l=43$.

Neste formato de ladrilho os polihexágonos construídos possuem apenas uma camada.

A bola do Tipo 1 é definida como $\tilde{B}_{r}=\left\{(a, 0) M_{l, 1} \in \mathbb{R}^{2}:(a, 0) G_{l, 1}(a, 0)^{T} \leq r^{2}<(l+\right.$ $1) / 2$ e $\left.(a, 0) \in \mathbb{Z}_{+}^{2}\right\}$, em que $G_{l, 1}$ é a matriz de Gram associada a $M_{l, 1}$ que gera $\Lambda_{a, l, 1}$. 
Teorema 4.1. Os polihexágonos do Tipo 1 ladrilham o $\mathbb{R}^{2}$.

Teorema 4.2. Para cada $l \in \mathbb{N}$ tal que $-l \equiv 1(\bmod 4)$, o número de raios para os quais temos códigos perfeitos do Tipo 1 em $\Lambda_{a, l, 1}$ é igual a $\lfloor\sqrt{l-3} / 2\rfloor$.

\subsubsection{Tipo 2: polihexágono de três níveis}

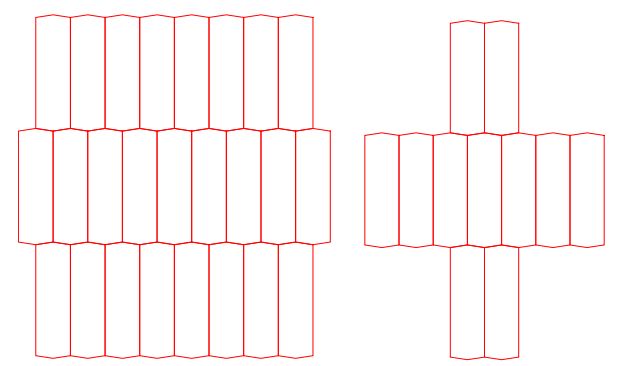

Figura 2: Polihexágonos do Tipo 2 construídos para $l=43$.

Nesse formato de ladrilho os polihexágonos possuem três camadas, sendo a camada central sempre maior do que as camadas laterais.

A bola $\tilde{B}_{r}$ do Tipo 2 é composta por todos os vetores $(a, 1) M_{l, 1},(b, 0) M_{l, 1} \in \mathbb{R}^{2}$ tais que $(b, 0) G_{l, 1}(b, 0)^{T} \leq r^{2}<2 l$ e $(a, 1) G_{l, 1}(a, 1)^{T} \leq r^{2}<2 l$, em que $G_{l, 1}$ é a matriz de Gram de $\Lambda_{a, l, 1}$. Definimos então a bola do Tipo 2 como $\tilde{B}_{r}=\left\{\boldsymbol{u} M_{l, 1} \in \mathbb{R}^{2}:(l+1) / 2 \leq \boldsymbol{u} G_{l, 1} \boldsymbol{u}^{T} \leq r^{2}<2 l\right.$ e $\boldsymbol{u} \in$ $\mathbb{Z}_{+}^{2}$, em que $\boldsymbol{u}=(a, 1)$ ou $\left.(b, 0)\right\}$.

Teorema 4.3. $\mathcal{R}_{1}$ é um $\Lambda_{2}$-ladrilho para $\mathbb{R}^{2}$

Teorema 4.4. Para cada $l \in \mathbb{N}$ tal que $-l \equiv 1(\bmod 4)$ temos que o número de raios para os quais $\Lambda_{l, 1} \subset \Lambda_{a, l, 1}$ é um código perfeito do Tipo 2 em que o raio da bola é dado por um vetor do tipo $(a, 1) M_{l, 1}$ é igual a $\lfloor(1+\sqrt{3 l}) / 2\rfloor$, se $3 l$ não é um quadrado perfeito, e $(1+\sqrt{3 l}) / 2-1$, caso contrário.

\section{$4.2 l \equiv 2(\bmod 4)$}

O reticulado algébrico $\Lambda_{a, l, 2}$ possui matriz geradora e matriz de Gram, respectivamente,

$$
M=\left(\begin{array}{cc}
1 & 1 \\
\sqrt{l} & -\sqrt{l}
\end{array}\right) \text { e } G=\left(\begin{array}{cc}
2 & 0 \\
0 & 2 l
\end{array}\right) .
$$

Note que $\operatorname{det}\left(\Lambda_{a, l, 2}\right)=4 l$ e a distância mínima $d$ é igual 2. Então, temos que

$$
\Delta\left(\Lambda_{a, l, 2}\right)=\frac{\pi(2 / 2)^{2}}{2 \sqrt{l}}=\frac{\pi}{2 \sqrt{l}} \Longrightarrow \Delta\left(\Lambda_{a, l, 2}\right) \longrightarrow 0, \text { quando } l \longrightarrow+\infty .
$$

Observe também que o raio de cobertura $R_{\Lambda_{a, l, 2}}$ é igual a $\sqrt{2 l+1} / 2$. Então,

$$
r \leq R_{\Lambda_{a, l, 2}} \frac{\left(1+\left(\Delta^{n}\right)^{1 / n}\right)}{\left(1-\left(\Delta^{n}\right)^{1 / n}\right)}=\frac{\sqrt{2 l+1}}{2} \frac{\left(1+(\pi / \sqrt{12})^{1 / 2}\right)}{\left(1-(\pi / \sqrt{12})^{1 / 2}\right)} .
$$

Logo, como $\frac{\sqrt{2 l+1}}{2} \frac{\left(1+(\pi / \sqrt{12})^{1 / 2}\right)}{\left(1-(\pi / \sqrt{12})^{1 / 2}\right)} \longrightarrow+\infty$ quando $l \longrightarrow+\infty$, quanto maior o valor de $l$, mais chances teremos de encontrar raios grandes para os quais existem códigos perfeitos em $\Lambda_{a, l, 2}$. 
Para essa família de reticulados, para cada valor de $l$, considerando os raios para os quais $\Lambda_{l, 2} \subset$ $\Lambda_{a, l, 2}$ é um código perfeito, também observamos que os poliominos construídos, que chamaremos aqui de poliretângulos, possuem alguns formatos específicos, e estudaremos três desses formatos, que denotaremos por "Tipo 1" e "Tipo 2". A seguir, vamos descrever esses formatos e fornecer alguns resultados sobre cada um deles.

\subsubsection{Tipo 1: poliretângulo de um nível}

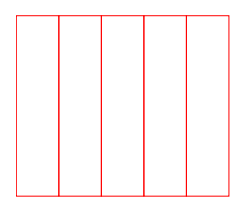

Figura 3: Poliretângulo do Tipo 1 construído para $l=18$.

Neste formato de ladrilho os poliretângulos construídos possuem apenas uma camada.

A bola do Tipo 1 é definida como $\tilde{B}_{r}=\left\{(a, 0) M_{l, 2} \in \mathbb{R}^{2}:(a, 0) G_{l, 2}(a, 0)^{T} \leq r^{2}<2 l\right.$ e $(a, 0) \in$ $\left.\mathbb{Z}_{+}^{2}\right\}$, em que $G_{l, 2}$ é a matriz de Gram de $\Lambda_{a, l, 2}$ associada a $M_{l, 2}$.

Teorema 4.5. Os poliretângulos do Tipo 1 ladrilham o $\mathbb{R}^{2}$.

Teorema 4.6. Para cada $l \in \mathbb{N}$ tal que $l \equiv 2(\bmod 4)$, o número de raios para os quais $\Lambda_{l, 2} \subset \Lambda_{a, l, 2}$ é um código perfeito do Tipo 1 é igual a $\lfloor\sqrt{l}\rfloor$.

\subsubsection{Tipo 2: poliretângulo de três níveis}

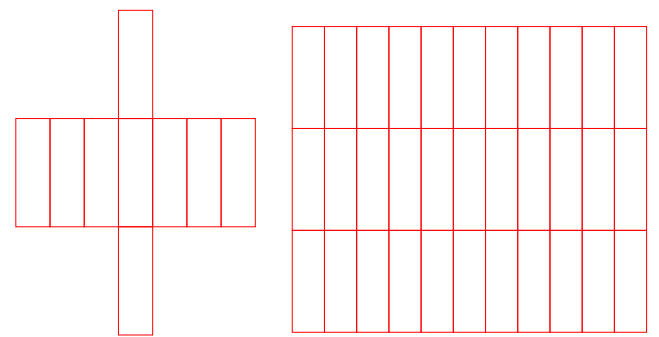

Figura 4: Poliretângulos do Tipo 2 construídos para $l=10$.

Neste formato de ladrilho os poliretângulos possuem três camadas, sendo a camada central maior ou igual do que as camadas laterais.

A bola $\tilde{B}_{r}$ do Tipo 2 é composta por todos os vetores $(a, 1) M_{l, 2},(b, 0) M_{l, 2} \in \mathbb{R}^{2}$ tais que $(a, 1) G_{l, 2}(a, 1)^{T} \leq r^{2}<2 l$ e $(b, 0) G_{l, 2}(b, 0)^{T} \leq r^{2}<2 l$. Definimos então a bola do Tipo 2 como $\tilde{B}_{r}=\left\{\boldsymbol{u} M_{l, 2} \in \mathbb{R}^{2}: 2 l \leq \boldsymbol{u} G_{l, 2} \boldsymbol{u}^{T} \leq r^{2}<8 l\right.$ e $\boldsymbol{u} \in \mathbb{Z}_{+}^{2}$, em que $\boldsymbol{u}=(a, 1)$ ou $\left.(b, 0)\right\}$.

Teorema 4.7. $\mathcal{R}_{1}$ é um $\Lambda_{2}$-ladrilho para $\mathbb{R}^{2}$.

Example Para $l=2$, os pares $(\alpha, \beta)$ que são poliretângulos do Tipo 2 são $(1,0),(1,1),(2,1)$ $e(2,2)$.

Teorema 4.8. Para cada $l \in \mathbb{N}$ tal que $l \equiv 2(\bmod 4)$, o número de raios para os quais $\Lambda_{l, 2} \subset \Lambda_{a, l, 2}$ é um código perfeito do Tipo 2 em que o raio da bola é dado por um vetor do tipo $(a, 1) M_{l, 2}$ é igual $a\lceil\sqrt{3 l}\rceil$. 


\section{Conclusões}

Em reticulados ambientes com raio de cobertura grande, vide reticulados algébricos apresentados, se mostram muito mais ricos que os reticulados $\mathbb{Z}^{2}$ e Hexagonal do ponto de vista da quantidade de Códigos Perfeitos.

\section{Agradecimentos}

Os autores agradecem à CAPES, ao CNPq (313326/2017-7, 141343/2019-2, 432735/2016-0 e 429346/2018-2) e a FAPESP (2013/25977-7).

\section{Referências}

[1] Campello, A., Jorge, G. C., Strapasson, J. E. and Costa, S. I. R., Perfect codes in the $l_{p}$ metric, European Journal of Combinatorics, 53:72-85, 2016. DOI:10.1016/j.ejc.2015.11.002.

[2] Conway, J. H. and Sloane, N. J. A. Sphere Packings, Lattices and Groups. New York: Springer Verlag, 3 ed., 1998.

[3] Costa, S. I. R., Muniz, M., Agustini, E. and Palazzo, R., Graphs, tessellations, and perfect codes on flat tori, IEEE Transactions on Information Theory, 50:2363-2377, 2004. DOI:10.1109/TIT.2004.834754.

[4] Etzion, T., Vardy, A. and Yaakobi, E., Dense error-correcting codes in the lee metric, IEEE Information Theory Workshop, 1-5, 2010. DOI:10.1109/CIG.2010.5592743.

[5] Golomb, S. W. and Welch, L. R. Perfect codes in the lee metric and the packing of polyominoes, SIAM Journal on Applied Mathematics, 18:302-317, 1970. DOI:10.1137/0118025.

[6] Horak, P., and AlBdaiwi, B. F., Diameter perfect lee codes, IEEE Transactions on Information Theory, 58:5490-5499, 2012. DOI:10.1109/TIT.2012.2196257.

[7] Kim, D., Nonexistence of perfect 2-error-correcting lee codes in certain dimensions, European Journal of Combinatorics, 63:1-5, 2017. DOI:10.1016/j.ejc.2017.01.007.

[8] Kovacevic, M., Codes in $A_{n}$ lattices: Geometry of $B_{h}$ sets and difference sets, arXiv 2016. arXiv:1409.5276.

[9] Livingston, M. and Q. F. Stout, Perfect dominanting sets, Congr. Numer., 79:187-203, 1990.

[10] Qureshi, C., Campello, A. and Costa, S. I. R., Non-existence of linear perfect lee codes with radius 2 for infinitely many dimensions, IEEE Transactions on Information Theory, 64:30423047, 2018. DOI:10.1109/TIT.2018.2797049.

[11] Strapasson, J. E., Jorge, G. C., Campello, A. and Costa, , Quasi-perfect codes in the $l_{p}$ metric, Computational and Applied Mathematics,37:852-866, 2018. DOI:10.1007/s40314-016-0372-2

[12] Strey, G., Campello, A., Strapasson, J. E. and Costa, S. I. R., Perfect Codes in Euclidean Lattices: Bounds and Case Studies, 2019 IEEE International Symposium on Information Theory (ISIT), Paris, France, 1607-1611, 2019. DOI:10.1109/ISIT.2019.8849538 